\title{
Arrêté du 1er octobre 1997 relatif aux bilans de la carte sanitaire de certaines installations ou activités de soins dont les besoins sont mesurés par un indice et pris pour l'application de l'article $R$. 712-39-1 du code de la santé publique (J.O. du 15-10-97, pp 14958-14960)
}

Le ministre de l'emploi et de la solidarité,

Arrête :

Art. 2. - Le bilan de la carte sanitaire des établissements autorisés à pratiquer les activités cliniques d'assistance médicale à la procréation est établi comme il apparaît en annexe II ci-jointe.

Art. 3. - Le bilan de la carte sanitaire des établissements autorisés à pratiquer les activités biologiques d'assistance médicale à la procréation est établi comme il apparaît en annexe III ci-jointe.

Art. 4. - Le bilan de la carte sanitaire des établissements autorisés à pratiquer les activités de diagnostic prénatal par les techniques de biochimie portant sur les marqueurs sériques d'origine embryonnaire et fotale dans le sang maternel est établi comme il apparaît en annexe IV ci-jointe.

Art. 5. - Conformément à l'article R. 712-39-1 du code de la santé publique, ces bilans seront publiés au Journal officiel de la République française. Ils seront affichés au siège des agences régionales de l'hospitalisation ainsi que dans les directions régionales et dans les directions départementales des affaires sanitaires et sociales. Cet affichage sera maintenu jusqu'au 31 décembre 1997.

Art. 6. - Le directeur des hôpitaux et les directeurs des agences régionales de l'hospitalisation sont chargés, chacun en ce qui le concerne, de l'exécution du présent arrêté, qui sera publié au Journal officiel de la République française. 


\section{BILAN DE LA CARTE SANITAIRE DES ETABLISSEMENTS AUTORISES A PRATIQUER LES ACTIVITES CLINIQUES D'ASSISTANCE MEDICALE A LA PROCREATION}

\begin{tabular}{|c|c|c|c|c|}
\hline $\begin{array}{l}\text { ZONES SANITAIRES : } \\
\text { REGIONS }\end{array}$ & BESOINS & $\begin{array}{l}\text { NOMBRE } \\
\text { de sites } \\
\text { autorisés }\end{array}$ & $\begin{array}{c}\text { BILAN } \\
+: \text { excédents } \\
-: \text { déficits } \\
0: \text { besoins } \\
\text { satisfaits }\end{array}$ & $\begin{array}{c}\text { DEMANDES } \\
\text { nouvelles } \\
\text { recevables }\end{array}$ \\
\hline Alsace & 3 & 2 & -1 & OUI \\
\hline Aquitaine & 5 & 5 & 0 & NON \\
\hline Auvergne & 2 & 2 & 0 & NON \\
\hline Bourgogne & 3 & 2 & -1 & OUI \\
\hline Bretagne & 5 & 5 & 0 & NON \\
\hline Centre & 4 & 3 & -1 & OUI \\
\hline Champagne-Ardenne & 3 & 3 & 0 & NON \\
\hline Corse & - & 0 & - & OUI \\
\hline Franche-Comté & 2 & 2 & 0 & NON \\
\hline Ile-de-France & 23 & 24 & +1 & NON \\
\hline Languedoc-Roussillon & 4 & 4 & 0 & NON \\
\hline Limousin & 1 & 1 & 0 & NON \\
\hline Lorraine & 4 & 4 & 0 & NON \\
\hline Midi-Pyrénées & 4 & 3 & -1 & OUI \\
\hline Nord-Pas-de-Calais & 7 & 3 & -4 & OUI \\
\hline Basse-Normandie & 2 & 1 & -1 & OUI \\
\hline Haute-Normandie & 3 & 3 & 0 & NON \\
\hline Pays de la Loire & 5 & 5 & 0 & NON \\
\hline Picardie & 3 & 2 & -1 & OUI \\
\hline Poitou-Charentes & 3 & 1 & -2 & OUI \\
\hline Provence-Alpes-Côte d'Azur & 8 & 7 & -1 & OUI \\
\hline Rhône-Alpes & 10 & 10 & 0 & NON \\
\hline Guadeloupe & 1 & 1 & 0 & NON \\
\hline Martinique & 1 & 1 & 0 & NON \\
\hline Guyane & - & 1 & 0 & NON \\
\hline Réunion & 1 & 2 & +1 & NON \\
\hline
\end{tabular}




\section{BILAN DE LA CARTE SANITAIRE DES ETABLISSEMENTS AUTORISES A PRATIQUER LES ACTIVITES BIOLOGIQUES D'ASSISTANCE MEDICALE A LA PROCREATION}

\begin{tabular}{|c|c|c|c|c|}
\hline $\begin{array}{l}\text { ZONES SANITAIRES : } \\
\text { REGIONS }\end{array}$ & BESOINS & $\begin{array}{l}\text { NOMBRE } \\
\text { de sites } \\
\text { autorisés }\end{array}$ & $\begin{array}{c}\text { BILAN } \\
+: \text { excédents } \\
-: \text { déficits } \\
0: \text { besoins } \\
\text { satisfaits }\end{array}$ & $\begin{array}{c}\text { DEMANDES } \\
\text { nouvelles } \\
\text { recevables }\end{array}$ \\
\hline Alsace & 3 & 3 & 0 & NON \\
\hline Aquitaine & 5 & 5 & 0 & NON \\
\hline Auvergne & 2 & 1 & -1 & OUI \\
\hline Bourgogne & 3 & 3 & 0 & NON \\
\hline Bretagne & 5 & 5 & 0 & NON \\
\hline Centre & 4 & 2 & -2 & OUI \\
\hline Champagne-Ardenne & 3 & 3 & 0 & NON \\
\hline Corse & - & 0 & - & OUI \\
\hline Franche-Comté & 2 & 2 & 0 & NON \\
\hline Ile-de-France & 23 & 22 & -1 & OUI \\
\hline Languedoc-Roussillon & 4 & 3 & -1 & OUI \\
\hline Limousin & 1 & 1 & 0 & NON \\
\hline Lorraine & 4 & 4 & 0 & NON \\
\hline Midi-Pyrénées & 4 & 3 & -1 & OUI \\
\hline Nord- Pas-de-Calais & 7 & 5 & -2 & OUI \\
\hline Basse-Normandie & 2 & 1 & -1 & OUI \\
\hline Haute-Normandie & 3 & 3 & 0 & NON \\
\hline Pays de la Loire & 5 & 5 & 0 & NON \\
\hline Picardie & 3 & 2 & -1 & OUI \\
\hline Poitou-Charentes & 3 & 1 & -2 & OUI \\
\hline Provence-Alpes-Côte d'Azur & 8 & 8 & 0 & NON \\
\hline Rhône-Alpes & 10 & 9 & -1 & OUI \\
\hline Guadeloupe & 1 & 1 & 0 & NON \\
\hline Martinique & 1 & 1 & 0 & NON \\
\hline Guyane & - & 1 & 0 & NON \\
\hline Réunion & 1 & 1 & 0 & NON \\
\hline
\end{tabular}




\section{ANNEXE IV}

\section{BILAN DE LA CARTE SANITAIRE DES ETABLISSEMENTS AUTORISES A PRATIQUER LES ACTIVITES DE DIAGNOSTIC PRENATAL PAR LES TECHNIQUES DE BIOCHIMIE PORTANT SUR LES MARQUEURS SERIQUES D'ORIGINE EMBRYONNAIRE ET FCETALE DANS LE SANG MATERNEL}

\begin{tabular}{|c|c|c|c|c|}
\hline $\begin{array}{l}\text { ZONES SANITAIRES : } \\
\text { REGIONS }\end{array}$ & BESOINS & $\begin{array}{l}\text { NOMBRE } \\
\text { de sites } \\
\text { autorisés }\end{array}$ & $\begin{array}{c}\text { BILAN } \\
+: \text { excédents } \\
\text { - : déficits } \\
0 \text { : besoins } \\
\text { satisfaits }\end{array}$ & $\begin{array}{c}\text { DEMANDES } \\
\text { nouvelles } \\
\text { recevables }\end{array}$ \\
\hline Alsace & 2 & 1 & -1 & OUI \\
\hline Aquitaine & 3 & 2 & -1 & OUI \\
\hline Auvergne & 1 & 1 & 0 & NON \\
\hline Bourgogne & 2 & 1 & -1 & OUI \\
\hline Bretagne & 3 & 3 & 0 & NON \\
\hline Centre & & 3 & 0 & NON \\
\hline Champagne-Ardenne & 2 & 1 & -1 & OUI \\
\hline Corse & - & 0 & - & OUI \\
\hline Franche-Comté & 1 & 0 & -1 & OUI \\
\hline Ile-de-France & 15 & 15 & 0 & NON \\
\hline Languedoc-Roussillon & 2 & 1 & -1 & OUI \\
\hline Limousin & 1 & 0 & -1 & OUI \\
\hline Lorraine & 3 & 3 & 0 & NON \\
\hline Midi-Pyrénées & 3 & 3 & 0 & NON \\
\hline Nord-Pas-de-Calais & 5 & 4 & -1 & OUI \\
\hline Basse-Normandie & 2 & 1 & -1 & OUI \\
\hline Haute-Normandie & 2 & 2 & 0 & NON \\
\hline Pays de la Loire & 4 & 4 & 0 & NON \\
\hline Picardie & 2 & 1 & -1 & OUI \\
\hline Poitou-Charentes & 2 & 0 & -2 & OUI \\
\hline Provence-Alpes-Côte d'Azur & 5 & 3 & -2 & OUI \\
\hline Rhône-Alpes & 6 & 5 & -1 & OUI \\
\hline Guadeloupe & - & 0 & - & OUI \\
\hline Martinique & 1 & 0 & -1 & OUI \\
\hline Guyane & - & 0 & - & OUI \\
\hline Réunion & 1 & 0 & -1 & OUI \\
\hline
\end{tabular}

